\title{
Characteristics of Hospitalized COVID-19 Patients Discharged and Experiencing Same-Hospital Readmission — United States, March-August 2020
}

\author{
Amy M. Lavery, $\mathrm{PhD}^{1}$; Leigh Ellyn Preston, DrPH ${ }^{1}$; Jean Y. Ko, $\mathrm{PhD}^{1}$; Jennifer R. Chevinsky, MD ${ }^{1}$; Carla L. DeSisto, PhD ${ }^{1}$; \\ Audrey F. Pennington, $\mathrm{PhD}^{1}$; Lyudmyla Kompaniyets, $\mathrm{PhD}^{1}$; S. Deblina Datta, MD ${ }^{1}$; Eleanor S. Click, MD, PhD ${ }^{1}$; Thomas Golden, MD ${ }^{1}$; \\ Alyson B. Goodman, MD ${ }^{1}$; William R. Mac Kenzie, $\mathrm{MD}^{1}$; Tegan K. Boehmer, $\mathrm{PhD}^{1}$; Adi V. Gundlapalli, MD, $\mathrm{PhD}^{1}$
}

On November 9, 2020, this report was posted as an MMWR Early Release on the MMWR website (https://www.cdc.gov/mmwr). Coronavirus disease 2019 (COVID-19) is a complex clinical illness with potential complications that might require ongoing clinical care (1-3). Few studies have investigated discharge patterns and hospital readmissions among large groups of patients after an initial COVID-19 hospitalization (4-7). Using electronic health record and administrative data from the Premier Healthcare Database, ${ }^{*}$ CDC assessed patterns of hospital discharge, readmission, and demographic and clinical characteristics associated with hospital readmission after a patient's initial COVID-19 hospitalization (index hospitalization). Among 126,137 unique patients with an index COVID-19 admission during March-July 2020, 15\% died during the index hospitalization. Among the 106,543 (85\%) surviving patients, $9 \%(9,504)$ were readmitted to the same hospital within 2 months of discharge through August 2020. More than a single readmission occurred among $1.6 \%$ of patients discharged after the index hospitalization. Readmissions occurred more often among patients discharged to a skilled nursing facility (SNF) (15\%) or those needing home health care $(12 \%)$ than among patients discharged to home or selfcare $(7 \%)$. The odds of hospital readmission increased with age among persons aged $\geq 65$ years, presence of certain chronic conditions, hospitalization within the 3 months preceding the index hospitalization, and if discharge from the index hospitalization was to a SNF or to home with health care assistance. These results support recent analyses that found chronic conditions to be significantly associated with hospital readmission $(6,7)$ and could be explained by the complications of underlying conditions in the presence of COVID-19 (8), COVID-19 sequelae (3), or indirect effects of the COVID-19 pandemic (9). Understanding the frequency of, and risk factors for, readmission can inform clinical practice, discharge disposition decisions, and public health priorities such as health care planning to ensure availability of resources needed for acute and follow-up care of COVID-19 patients. With the recent

\footnotetext{
*The Premier Healthcare Database includes discharge records for adult and pediatric patients from $>1,000$ nongovernmental, teaching and community hospitals representing approximately $25 \%$ of U.S. hospital admissions. Data for this study represented a subset of 865 medical facilities that contributed inpatient encounters to the Premier Healthcare Database during March-August 2020. https:/www.premierinc.com/newsroom/category/education/page/2.
}

increases in cases nationwide, hospital planning can account for these increasing numbers along with the potential for at least $9 \%$ of patients to be readmitted, requiring additional beds and resources.

Data for this study were obtained from the Premier Healthcare Database, which includes discharge records from 865 nongovernmental, community, and teaching hospitals that contributed inpatient data during the study period. COVID-19 patients were identified through International Classification of Diseases, Tenth Revision, Clinical Modification (ICD-10-CM) discharge diagnosis code of U07.1 (COVID-19, virus identified) during April-July 2020 or B97.29 (Other coronavirus as the cause of disease classified elsewhere [recommended before the April 2020 release of U07.1 $]^{\dagger}$ ) during March-April 2020. Both codes were used for discharges during April. The patient's first hospitalization with a COVID-19 discharge diagnosis was defined as the index hospitalization. Any subsequent hospitalization occurring within 2 months of the index hospitalization discharge date through August 2020, whether for COVID-19 or other health complications, was considered a hospital readmission. ${ }^{\S}$ Hospital readmissions that occurred $>2$ months after the index hospitalization were excluded. In the Premier Healthcare Database, readmissions were only recorded if a patient returned to the same hospital where the index hospitalization occurred.

Demographic and clinical characteristics of patients at their index hospitalization were compared regarding discharge disposition and readmission status (none versus one or more). Presence of selected chronic conditions associated with a more severe COVID-19 clinical course were identified through ICD-10-CM diagnosis codes during or before the index COVID-19 hospitalization. Visits before the index hospitalization included all inpatient encounters for the cohort during calendar year 2020 only. Five chronic conditions that have been identified by CDC to increase or possibly increase the risk for severe COVID-19-associated illness (chronic obstructive pulmonary disease, heart failure, diabetes [type 1 or type 2 , with chronic complications], chronic kidney disease, and obesity [body mass index $\geq 30 \mathrm{~kg} / \mathrm{m}^{2}$ ], including

\footnotetext{
${ }^{\dagger}$ https://www.cdc.gov/nchs/data/icd/Announcement-New-ICD-code-forcoronavirus-3-18-2020.pdf.

${ }^{\$}$ Two months was twice the period used by the Centers for Medicare \& Medicaid Services for unplanned readmission measures as knowledge of COVID-19 has been evolving regarding acute and chronic sequelae.
} 
severe obesity, [body mass index $\geq 40 \mathrm{~kg} / \mathrm{m}^{2}$ ]) were mapped to ICD-10-CM codes using the Elixhauser Comorbidity Index (a method for classifying comorbidities based on ICD diagnosis codes found in administrative data; each comorbidity category is dichotomous [present or absent]) and implemented with the Elixhauser Comorbidity Software for ICD-10-CM (beta version; Agency for Healthcare Research and Quality) and R software (version 4.0.92020; The R Foundation) (10). The following three clinical severity indicators were defined using hospital chargemaster records (i.e., the comprehensive list of all items billable to a hospital patient or to a patient's insurance provider): intensive care unit (ICU) admission, invasive mechanical ventilation, and noninvasive ventilation. Time to readmission after the index hospitalization was calculated as the difference in days between date of readmission and date of discharge from the previous hospitalization. The primary discharge diagnosis for each hospitalization was categorized into Clinical Classification Software Refined Categories to approximate the primary reason for the hospital stay. A multivariable generalized estimating equation model assessed the odds of readmission, accounting for within-facility correlation. Covariates included in the model were age, sex, race/ethnicity, presence of selected chronic conditions, discharge disposition category, and clinical severity indicators. This activity was reviewed by CDC and was conducted consistent with applicable federal law and CDC policy.**

During March-July 2020, a total of 126,137 patients within the Premier Healthcare Database were hospitalized for COVID-19. The majority of patients were admitted from a non-health care setting $(81 \%)$, followed by transfer from another hospital, clinic, or SNF (18\%) (Table 1). During the index hospitalization, $15 \%$ of patients were admitted to an ICU, 13\% required invasive mechanical ventilation, and $4 \%$ required noninvasive ventilation. At the time of the index hospitalization or at any time during 2020 before the hospitalization, $62 \%$ of patients had an ICD-10-CM diagnosis code for one or more of the following five chronic conditions: chronic obstructive pulmonary disease (21\%), heart failure (16\%), diabetes mellitus type 1 or type $2(27 \%)$, chronic kidney disease $(21 \%)$, or obesity $(27 \%)$. Overall, $10,008(8 \%)$ patients had been hospitalized at the same hospital in the 3 months preceding their index COVID-19 hospitalization. Approximately $15 \%$ of patients $(19,594)$ died during the index hospitalization.

Among the 106,543 patients discharged from the index admission, 9,504 (9\%) were readmitted, including 1,667 $(1.6 \%)$ who were readmitted more than once. The median

\footnotetext{
https:/www.cdc.gov/coronavirus/2019-ncov/need-extra-precautions/peoplewith-medical-conditions.html.

** 45 C.F.R. part 46, 21 C.F.R. part 56; 42 U.S.C. Sect. 241(d); 5 U.S.C. Sect. 552a; 44 U.S.C. Sect. 3501 et seq.
}

interval from discharge to first readmission was 8 days (interquartile range $=3-20$ days). Less than $0.1 \%$ of patients died during readmission (data suppressed for privacy).

Among all patients who were discharged after the index hospitalization, $60 \%$ were discharged to home or self-care (to home without any additional professional services provided such as home nursing health care), $15 \%$ to a SNF, $10 \%$ to home with assistance from a home health organization, $4 \%$ to hospice, $4 \%$ to ongoing care, and $5 \%$ to other locations (Table 2). Readmission was more common among patients discharged to a SNF (15\%) or with home health organization support $(12 \%)$, compared with patients discharged to home or self-care (7\%). Median age, severity markers, time to readmission and length of stay differed by index hospitalization discharge disposition category.

When controlling for covariates, the odds of readmission increased with the presence of chronic obstructive pulmonary disease $(\mathrm{OR}=1.4)$, heart failure $(\mathrm{OR}=1.6)$, diabetes $(\mathrm{OR}=1.2)$, and chronic kidney disease $(\mathrm{OR}=1.6)$. Patients were more likely to be readmitted if they had been discharged from the index hospitalization to a SNF $(\mathrm{OR}=1.4)$ or with home health organization support $(\mathrm{OR}=1.3)$ than if they had been discharged to home or self-care. Compared with persons aged 18-39 years, the odds of readmission increased with age among persons aged $\geq 65$ years (Table 3 ). Adjusted odds of readmission of patients with a hospitalization in the 3 months preceding their index hospitalization were 2.6 times the odds of those who were not hospitalized in the preceding 3 months. Non-Hispanic White persons were more likely to be readmitted than were those of other racial/ethnic groups. Common primary discharge diagnoses after readmission were infectious and parasitic diseases (primarily COVID-19; 45\%) and diseases of the circulatory (11\%) and digestive (7\%) systems (Supplementary Table, https://stacks.cdc.gov/view/cdc/96391).

\section{Discussion}

In a cohort of 106,543 patients discharged after an index COVID-19 hospitalization, 9\% experienced at least one readmission to the same hospital within 2 months of discharge. More than one readmission occurred in $1.6 \%$ of cases. In this analysis, the odds of hospital readmission increased with age among persons aged $\geq 65$ years, presence of one of five selected chronic conditions, hospitalization within the 3 months preceding the index hospitalization, and if discharge from the index hospitalization was to a SNF or to home with health care assistance. Although the proportions of patients in the Premier Healthcare Database cohort who were non-Hispanic Black (23\%) or Hispanic $(21 \%)$ were higher than those proportions in the U.S. Census (13\% and 18\%, respectively), their odds of readmission were lower than those of non-Hispanic White 
TABLE 1. Demographic characteristics of hospitalized COVID-19 patients at index hospitalization, by readmission status — Premier Healthcare Database, United States, March-August 2020

\begin{tabular}{|c|c|c|c|}
\hline \multirow[b]{3}{*}{ Characteristic } & \multicolumn{3}{|c|}{ No. (\%) } \\
\hline & Total & Not readmitted & Readmitted at least once \\
\hline & $N=126,137$ & $N=116,633$ & $\mathrm{~N}=9,504$ \\
\hline \multicolumn{4}{|l|}{ Age group (yrs) } \\
\hline$<18$ & $1,170(0.9)$ & $1,095(0.9)$ & $75(0.8)$ \\
\hline 18-39 & $16,699(13.2)$ & $15,741(13.5)$ & $958(10.1)$ \\
\hline $40-49$ & $14,490(11.5)$ & $13,674(11.7)$ & $816(8.6)$ \\
\hline $50-64$ & $35,451(28.1)$ & $32,923(28.2)$ & $2,528(26.6)$ \\
\hline $65-74$ & $25,419(20.2)$ & $23,250(19.9)$ & $2,169(22.8)$ \\
\hline $75-84$ & 19,864 (15.7) & $18,061(15.5)$ & 1,803 (19.0) \\
\hline$\geq 85$ & $13,044(10.3)$ & $11,889(10.2)$ & $1,155(12.2)$ \\
\hline \multicolumn{4}{|l|}{ Race/Ethnicity } \\
\hline Asian, non-Hispanic & $3,652(2.9)$ & $3,429(2.9)$ & $223(2.4)$ \\
\hline Black, non-Hispanic & $29,226(23.2)$ & $26,819(23.0)$ & $2,407(25.3)$ \\
\hline Hispanic & $26,921(21.3)$ & $25,412(21.8)$ & $1,509(15.9)$ \\
\hline White, non-Hispanic & $49,133(39.0)$ & $44,807(38.4)$ & $4,326(45.5)$ \\
\hline Other & $13,048(10.3)$ & $12,194(10.5)$ & $854(9.0)$ \\
\hline \multicolumn{4}{|l|}{ Sex } \\
\hline Female & $60,426(47.9)$ & $55,827(47.9)$ & 4,599 (48.4) \\
\hline Male & $65,597(52.0)$ & $60,695(52.0)$ & $4,902(51.6)$ \\
\hline Unknown & $114(0.1)$ & $111(0.1)$ & - $^{*}$ \\
\hline \multicolumn{4}{|l|}{ Point of origin } \\
\hline Non-health care & $102,482(81.2)$ & $94,796(81.3)$ & $7,686(74.5)$ \\
\hline Clinic & $6,787(5.4)$ & $6,217(5.3)$ & $570(5.5)$ \\
\hline Transfer from a different hospital & $8,425(6.7)$ & $7,968(6.8)$ & $457(4.4)$ \\
\hline Transfer from SNF or ICF & $5,940(4.7)$ & $5,324(4.6)$ & $616(6.0)$ \\
\hline Transfer from health facility & $1,437(1.1)$ & $1,339(1.1)$ & $98(1.0)$ \\
\hline Court/Law enforcement & $252(0.2)$ & $241(0.2)$ & $11(0.1)$ \\
\hline Born inside the hospital & $45(0.0)$ & $44(0.0)$ & - ${ }^{*}$ \\
\hline Not available & $441(0.3)$ & $410(0.4)$ & $31(0.3)$ \\
\hline \multicolumn{4}{|l|}{ U.S. Census division } \\
\hline East North Central & $16,009(12.7)$ & $14,547(12.5)$ & $1,462(15.4)$ \\
\hline East South Central & $5,986(4.7)$ & $5,544(4.8)$ & $442(4.7)$ \\
\hline Middle Atlantic & $39,673(31.5)$ & $36,456(31.3)$ & $3,217(33.9)$ \\
\hline Mountain & $8,852(7.0)$ & $8,355(7.2)$ & $497(5.2)$ \\
\hline New England & $3,768(3.0)$ & $3,346(2.9)$ & $422(4.4)$ \\
\hline Pacific & $6,511(5.2)$ & $6,138(5.3)$ & $373(3.9)$ \\
\hline South Atlantic & $27,407(21.7)$ & $25,683(22.0)$ & $1,724(18.1)$ \\
\hline West North Central & $4,364(3.5)$ & $3,998(3.4)$ & 366 (3.9) \\
\hline West South Central & $13,567(10.8)$ & $12,566(10.8)$ & $1,001(10.5)$ \\
\hline
\end{tabular}

Abbreviations: COVID-19 = coronavirus disease 2019; ICF = intermediate care facility; SNF = skilled nursing facility.

* Cell sizes $<10$ were suppressed.

patients. The slight association of readmission with lengths of stay for hospitalized COVID-19 patients merits further study.

These results are comparable to those of recently published analyses, which found a similar group of chronic conditions to be significantly associated with hospital readmission $(6,7)$ and could be explained by the complications of underlying conditions in the presence of COVID-19 (8), COVID-19 sequelae (3), or indirect effects of the COVID-19 pandemic (9). Although only a small proportion of patients discharged to home or self-care were readmitted, 7\% returned to the hospital within a median of 7 days. One explanation for their readmission is that approximately two thirds of these 4,406 patients had one or more of the selected chronic conditions.
After hospitalization for COVID-19, the most common primary discharge diagnoses from hospital readmission were diseases of the circulatory, digestive, or respiratory systems. Future work will examine the detailed diagnoses recorded during readmissions to better understand COVID-19 sequelae or health conditions that require extended or ongoing care.

The findings in this report are subject to at least five limitations. First, COVID-19 diagnoses were determined by ICD-10-CM, not through laboratory confirmation, potentially leading to misclassification of cases. Second, chronic conditions were identified using ICD-10-CM diagnostic codes used at the index hospitalization or a previous encounter. If a patient had a chronic condition but the condition was not assigned a diagnostic code, that condition would not be recorded in this 
TABLE 2. Discharge status and subsequent readmissions among 126,137 COVID-19 patients* with an index hospitalization — United States, March-August 2020

Location to which patient was discharged from index hospitalization

\begin{tabular}{|c|c|c|c|c|c|c|}
\hline \multirow[b]{2}{*}{ Characteristic } & \\
\hline & Home or self-care & SNF & $\begin{array}{l}\text { Home health } \\
\text { organization }\end{array}$ & Hospice & Ongoing care $^{\dagger}$ & Other $\S$ \\
\hline \multicolumn{7}{|l|}{ Discharged $(\mathrm{N}=106,543[85 \%])$} \\
\hline No. of patients discharged, (\%) & $64,475(60)$ & $16,339(15)$ & $12,223(10)$ & $3,807(4)$ & $4,404(4)$ & $5,295(5)$ \\
\hline Length of index hospitalization, days, median (IQR) & $4(2-7)$ & $8(5-15)$ & $8(4-14)$ & $7(4-12)$ & $16(7-29)$ & $3(1-7)$ \\
\hline Male, \% & 51 & 47 & 49 & 47 & 57 & 61 \\
\hline Median age, yrs & 53 & 76 & 68 & 83 & 66 & 61 \\
\hline$\geq 1$ chronic condition, $\%$ & 53 & 72 & 70 & 67 & 70 & 57 \\
\hline ICU admission, \% & 35 & 42 & 45 & 53 & 63 & 42 \\
\hline \multicolumn{7}{|l|}{ Readmitted $(\mathrm{N}=9,504,9 \%)^{\uparrow}$} \\
\hline No. (\%) of patients readmitted & $4,406(7)$ & $2,517(15)$ & $1,469(12)$ & $136(4)$ & $494(11)$ & $482(9)$ \\
\hline No. days to readmission, median (IQR) & $7(3-17)$ & $11(5-25)$ & $8(3-19)$ & $0(0-3)$ & $10(3-25)$ & $6(1-21)$ \\
\hline Length of hospitalization, days, median (IQR) & $4(2-7)$ & $6(3-9)$ & $5(3-8)$ & $3(1-6)$ & $6(3-10)$ & $4(2-8)$ \\
\hline Male, \% & 51 & 50 & 49 & 51 & 62 & 64 \\
\hline Median age, yrs & 58 & 75 & 72 & 80 & 67 & 59 \\
\hline$\geq 1$ chronic condition, $\%$ & 67 & 80 & 80 & 75 & 77 & 67 \\
\hline
\end{tabular}

Abbreviations: COVID-19 = coronavirus disease 2019; ICU = intensive care unit; IQR = interquartile range; SNF = skilled nursing facility.

* A total of 19,594 (15\%) patients died during the index hospitalization; $59 \%$ of decedents were male, median age was 74 years, $75 \%$ had one or more chronic conditions, the median hospitalization duration was 8 days (IQR $=4-15$ days), and $68 \%$ of patients were admitted to an ICU.

† Ongoing care categories include discharged/transferred to cancer center, admitted as an inpatient to this hospital, still a patient, discharged/transferred to federal hospital, discharged/transferred to swing bed unit (a unit within an acute care hospital where patients receive the same skilled level of care that is available at skilled nursing facilities), discharged/transferred to another rehabilitation facility, discharged/transferred to long-term care hospitals that provide acute inpatient care with an average length of stay of $\geq 25$ days, discharged to a psychiatric hospital, discharged/transferred to a critical access hospital.

$\S$ Other category includes patients who were discharged to other facilities and those who left against medical advice.

" Readmitted from discharged location noted in column (after index hospitalization).

\section{Summary}

What is already known about this topic?

Evidence suggests that potential health complications after COVID-19 illness might require ongoing clinical care.

What is added by this report?

After discharge from an initial COVID-19 hospitalization, $9 \%$ of patients were readmitted to the same hospital within 2 months of discharge. Multiple readmissions occurred in $1.6 \%$ of patients. Risk factors for readmission included age $\geq 65$ years, presence of certain chronic conditions, hospitalization within the 3 months preceding the first COVID-19 hospitalization, and discharge to a skilled nursing facility or with home health care.

What are the implications for public health practice?

Understanding frequency of, and potential reasons for, readmission after a COVID-19 hospitalization can inform clinical practice, discharge disposition decisions, and public health priorities, such as health care resource planning.

analysis. Third, primary discharge diagnosis was used to infer the primary reason for hospital admission; other diagnoses might have contributed to the reason for index admission and readmissions. Fourth, patients who received care at different hospitals would not be assessed longitudinally. Finally, the sequelae of COVID-19 could not be completely described among hospitalized patients or among those readmitted.
Sequelae might be experienced by patients who are never readmitted to a hospital.

Information on the frequency of, and risk factors for, readmission can inform clinical practice and discharge disposition decisions especially with regard to the acuity and location of ongoing care needed for persons who might appear stable at discharge. Further, addressing priorities such as health care planning to ensure adequate health care resources for acute and post-acute follow-up care of COVID-19 patients is critical at a local, regional, and national level. With the recent increase in cases nationwide, hospital planning can account for these increasing numbers along with the potential for at least $9 \%$ of patients to be readmitted, requiring additional beds and resources. Continued public health messaging and interventions to prevent COVID-19 among older persons and those with underlying medical conditions is essential.

\section{Acknowledgments}

John House, Premier Inc.; Yulia Carroll, Melissa Danielson, Hussain Yusuf; members of the CDC COVID-19 Response Data, Analytics, and Modeling Task Force.

Corresponding author: Adi V. Gundlapalli, agundlapalli@cdc.gov.

${ }^{1}$ CDC COVID-19 Response Team.

All authors have completed and submitted the International Committee of Medical Journal Editors form for disclosure of potential conflicts of interest. No potential conflicts of interest were disclosed. 
TABLE 3. Generalized estimating equation model showing the adjusted odds of readmission among persons hospitalized with COVID-19 - United States, March-August 2020

\begin{tabular}{|c|c|c|c|}
\hline Characteristic & $\begin{array}{c}\text { Odds ratio } \\
(95 \% \mathrm{Cl})\end{array}$ & $\begin{array}{c}\text { Standard } \\
\text { error* }\end{array}$ & P-value \\
\hline \multicolumn{4}{|l|}{ Age group (yrs), (referent $=18-39$ yrs) } \\
\hline$<18$ & $1.03(0.80-1.33)$ & 0.13 & 0.806 \\
\hline $40-49$ & $0.94(0.84-1.04)$ & 0.05 & 0.204 \\
\hline $50-64$ & $1.08(0.99-1.17)$ & 0.04 & 0.078 \\
\hline $65-74$ & $1.22(1.12-1.34)$ & 0.05 & $<0.001$ \\
\hline $75-84$ & $1.32(1.20-1.46)$ & 0.05 & $<0.001$ \\
\hline$\geq 85$ & $1.37(1.23-1.53)$ & 0.06 & $<0.001$ \\
\hline \multicolumn{4}{|c|}{ Race/Ethnicity (referent = White, non-Hispanic) } \\
\hline Asian, non-Hispanic & $0.82(0.71-0.95)$ & 0.07 & 0.007 \\
\hline Black, non-Hispanic & $0.90(0.85-0.95)$ & 0.03 & $<0.001$ \\
\hline Hispanic & $0.75(0.71-0.81)$ & 0.03 & $<0.001$ \\
\hline Other & $0.80(0.74-0.87)$ & 0.04 & $<0.001$ \\
\hline \multicolumn{4}{|l|}{ Sex (referent = male) } \\
\hline Female & $0.94(0.90-0.99)$ & 0.02 & 0.015 \\
\hline \multicolumn{4}{|l|}{ Chronic conditions } \\
\hline COPD & $1.35(1.28-1.42)$ & 0.03 & $<0.001$ \\
\hline Heart failure & $1.58(1.48-1.67)$ & 0.03 & $<0.001$ \\
\hline Diabetes & $1.21(1.14-1.28)$ & 0.03 & $<0.001$ \\
\hline Chronic kidney disease & $1.64(1.55-1.74)$ & 0.03 & $<0.001$ \\
\hline Obesity & $0.95(0.90-1.00)$ & 0.03 & 0.049 \\
\hline Previous hospitalization ${ }^{\dagger}$ (yes versus no) & $2.61(2.45-2.78)$ & 0.03 & $<0.001$ \\
\hline \multicolumn{4}{|c|}{ Severity measures at index hospitalization } \\
\hline Length of stay, days & $0.99(0.99-1.00)$ & 0.00 & 0.001 \\
\hline ICU admission & $0.94(0.89-0.99)$ & 0.03 & 0.014 \\
\hline Mechanical ventilation & $1.15(1.04-1.27)$ & 0.05 & 0.006 \\
\hline Noninvasive ventilation & $0.86(0.81-0.90)$ & 0.03 & $<0.001$ \\
\hline \multicolumn{4}{|c|}{ Discharge category from index hospitalization (referent $=$ home/self-care) } \\
\hline SNF & $1.37(1.29-1.47)$ & 0.03 & $<0.001$ \\
\hline Home health organization & $1.30(1.21-1.39)$ & 0.04 & $<0.001$ \\
\hline Hospice & $0.24(0.20-0.29)$ & 0.09 & $<0.001$ \\
\hline Ongoing care & $1.22(1.09-1.36)$ & 0.06 & 0.001 \\
\hline
\end{tabular}

Abbreviations: $\mathrm{Cl}=$ confidence interval; $\mathrm{COPD}=$ chronic obstructive pulmonary disease; COVID-19 = coronavirus disease 2019; ICU = intensive care unit; SNF = skilled nursing facility.

* Standard error of coefficient.

† Patients who had a hospitalization within 3 months before their COVID-19 index hospitalization.

\section{References}

1. Bikdeli B, Madhavan MV, Jimenez D, et al.; Global COVID-19 Thrombosis Collaborative Group, Endorsed by the ISTH, NATF, ESVM, and the IUA, Supported by the ESC Working Group on Pulmonary Circulation and Right Ventricular Function. COVID-19 and thrombotic or thromboembolic disease: implications for prevention, antithrombotic therapy, and follow-up: JACC state-of-the-art review. J Am Coll Cardiol 2020;75:2950-73. PMID:32311448 https://doi. org/10.1016/j.jacc.2020.04.031

2. Whittaker A, Anson M, Harky A. Neurological manifestations of COVID-19: a systematic review and current update. Acta Neurol Scand 2020;142:14-22. PMID:32412088 https://doi.org/10.1111/ane.13266

3. Liu PP, Blet A, Smyth D, Li H. The science underlying COVID-19: implications for the cardiovascular system. Circulation 2020;142:68-78. PMID:32293910 https://doi.org/10.1161/ CIRCULATIONAHA.120.047549

4. Somani SS, Richter F, Fuster V, et al.; Mount Sinai COVID Informatics Center. Characterization of patients who return to hospital following discharge from hospitalization for COVID-19. J Gen Intern Med 2020;35:2838-44. PMID:32815060 https://doi.org/10.1007/ s11606-020-06120-6

5. Rokadiya S, Gil E, Stubbs C, Bell D, Herbert R. COVID-19: outcomes of patients with confirmed COVID-19 re-admitted to hospital. J Infect 2020;81:e18-9. PMID:32652166 https://doi.org/10.1016/j.jinf.2020.07.007

6. Jeon WH, Seon JY, Park SY, Oh IH. Analysis of risk factors on readmission cases of COVID-19 in the Republic of Korea: using nationwide health claims data. Int J Environ Res Public Health 2020;17:5844. PMID:32806775 https://doi.org/10.3390/ijerph17165844

7. Atalla E, Kalligeros M, Giampaolo G, Mylona EK, Shehadeh F, Mylonakis E. Readmissions among patients with COVID-19. Int J Clin Pract 2020;00:e13700. PMID:32894801 https://onlinelibrary.wiley. com/doi/10.1111/ijcp.13700

8. Bornstein SR, Rubino F, Khunti K, et al. Practical recommendations for the management of diabetes in patients with COVID-19. Lancet Diabetes Endocrinol 2020;8:546-50. PMID:32334646 https://doi. org/10.1016/S2213-8587(20)30152-2

9. Masroor S. Collateral damage of COVID-19 pandemic: delayed medical care. J Card Surg 2020;35:1345-7. PMID:32419177 https://doi. org/10.1111/jocs. 14638

10. Elixhauser A, Steiner C, Harris DR, Coffey RM. Comorbidity measures for use with administrative data. Med Care 1998;36:8-27. PMID:9431328 https://doi.org/10.1097/00005650-199801000-00004 